29

\section{IMPACT OF EARLY AND CONSISTENT OCCUPATIONAL THERAPY WITH PRETERM INFANTS' ORAL FEEDINGS.}

NM Mohr, SR Lacey, S Teasley, HW Kilbride, Department of Physical and Occupational Therapy, Children's Mercy Hospital and Clinics, Kansas City, Missour.

Purpose: This study was designed to assess the effectiveness of early occupational therapy intervention on preterm infant's ability to achieve full oral feedings and be discharged from the neonatal intensive care unit. Study design and methods: Forty-one preterm infants were randomized to receive occupational therapy intervention or sham intervention beginning at 30 weeks' gestational age. An oral stimulation and therapeutic feeding protocol was used for those infants in the experimental group. Sham intervention was used for the infants in the control group and included holding the infant without any provision of oral stimulation or therapeutic feedings. These interventions wer provided three times a week for 10-15 minutes each. Outcome measures included: postmenstrual age at first successful feeding, postmenstrual age at full oral feedings, number of infants receiving occupational therapy consultations, postmenstrual age at discharge from neonatal intensive care unit, and total length of hospital stay. Results: No significant differences were found in the aggregate; however, significant differences were found in the subgroup of less then 29 weeks' gestational age. Extremely preterm infants receiving early occupational therapy achieved full oral feedings on average 2.3 weeks earlier than their control counterparts. Conclusion: Early limited occupational therapy intervention may accelerate time to full oral feeding in extremely preterm infants.

\section{0}

INITIATION OF ORAL FEEDINGS IN INFANTS LESS THAN 34 WEEKS TO FACILITATE EARLY DISCHARGE FROM NICU.

K. Macwan, M. Shareef, V. Albert, D. Drenckpohl, Division of Neonatology, Department of Pediatrics, UIC College of Medicine at Peoria and Children's Hospital of Illinois at OSF Saint Francis Medical Center, Peoria, IL.

Background: Although the component of sucking and swallowing is present in the premature neonates at 28 weeks of gestation, the synchrony is irregular and oral feeding is difficult. The oral feedings become coordinated by $32-34$ weeks of gestation and so the premature neonates are usually offered oral feeding at 32-34 weeks corrected gestational age. We had several neonates who were discharge home at $\leq 34$ weeks of gestation. In order for them to be discharged at $\leq 34$ weeks of gestation age, they must have had their oral feedings started earlier then 32 weeks of corrected age. Retrospective study was done to find out the earliest gestational age at which it would be safe to initiate oral feedings for early discharge from NICU. Methods: We reviewed all the charts for the neonates oral feedings for early discharge from NICU. Methods: We reviewed all the charts for the neonates
who were discharge home from our neonatal intensive care unit (NICU) at $\leq 34$ weeks corrected gestational age from October 1, 2001 to December 31,2002 . The data was collected for gestational age, birth weight, sex, corrected gestational age, day of life the oral feedings were initiated, discharge weight and length of stay. Results: We had total 50 patients who were discharged home during the study period who were $\leq 34$ weeks of corrected gestational age. 0 Conclusion: Oral feedings can be

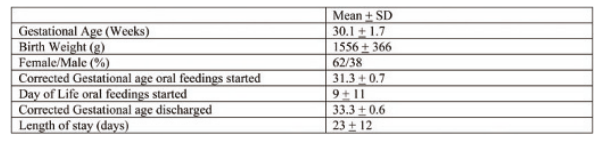

initiated as early as 30 weeks of corrected gestational age in neonates. Early initiation of oral feeding may shorten the length of stay in NICU.

\section{1}

THE LONG-TERM OUTCOME OF FUNDOPLICATION IN CHILDREN WITH GASTROESOPHAGEAL REFLUX DISEASE: A RETROSPECTIVE ANALYSIS.

H.Mousa, F.Woodley, M.Metheney, J.Hayes*, Division of Gastroenterology, Center for Biostatistics*, Ohio State University, Columbus Children's Hospital, Columbus, Ohio

Purpose of the Study: To evaluate our 5-year experience with anti-reflux surgery (ARS) and determine the parents' perspective of the outcome. Methods Used: We conducted a retrospective analysis of the outcome and quality of life as a result of anti-reflux surgery (ARS) performed at Columbus Children's Hospital during 1998-2003. Charts were reviewed for demographic data and surveys were sent out to 491 patients who underwent fundoplication, 119 were returned as addres unknown, 65 people returned the survey for a response rate of $17 \%(65 / 372)$ and 4 expressed the desire not to participate. We assessed the outcome and quality of life on 61 patients; age (mean 4 years, median 1), $37 \mathrm{M} / 24 \mathrm{~F}, 75 \%$ Caucasian. The primary reason for ARS was FTT n=11 (18\%), breathing problems $n=25(41 \%)$, and vomiting $n=23(38 \%)$. Nissen was performed on 60 and Thal on 1 .

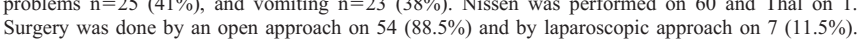
Among the 61 patients, 21(34\%) had neurological developmental delay. Results: Six months after ARS, $32 \%$ of patients ate exclusively by mouth compared to $55 \%$ prior to surgery $(p=0.001 *)$ and $22 \%$ remained on exclusive continuous jejunal feeding compared to $37 \%$ prior to ARS ( $p=0.09$ ) General overall health score, emotional state and respiratory condition were scored as (poor, fair, good, very good and excellent). Analysis of pre and post ARS values per Wilcox matched pair test revealed significant improvements with $\mathrm{p}<0.001$ in all 3 domains. Limitation of child's activity was scored significant improvements with $\mathrm{p}<0.001$ in all 3 domains. Limitation of child's activity was scored
between none and severe. Statistical analysis revealed significant improvement $(\mathrm{p}=0.001)$. Average number of workdays/month missed due to doctor visits was 19.2 compared to 8 post ARS $(\mathrm{p}=0.022)$. Conclusions: 1. Anti-reflux surgery does not improve the oral intake of children with GER. 2. Mos children fed via transpyloric access will continue to require this means of feeding after ARS. General quality of life improves after ARS and health care utilization decreases.
32

FACTORS INFLUENCING PARENT ANXIETY LEVELS IN A PEDIATRIC EMERGENCY DEPARTMENT WAITING AREA.

L Holm, L Fitzmaurice, Division of Pediatric Emergency Medicine, Children's Mercy Hospital, Kansas City, MO

Purpose: Bringing a sick child to the emergency department (ED) is inherently stressful. This stress is compounded by the ED waiting area environment, which can be loud and chaotic. The aim of this study was to determine the general stress level of adults accompanying children to a pediatric emergency department (ED). A secondary aim was to determine what factors are associated with these stress levels, with a focus on waiting area factors. Methods: This descriptive study was conducted with stress levels, with a focus on waiting area factors. Methods: This descriptive study was conducted with
a convenience sample of adults accompanying children to the ED over 7 consecutive days, with study blocks in the daytime and nighttime. Stress levels were measured using the Spielberger state anxiety inventory (STAI). The test is scored from 20 to 80 and has a published mean of 36 in working adult under normal conditions. College students in an experimental exam situation scored a mean of 43 . Additional questions eliciting the influence of other, especially environmental factors were asked. Demographic data as well as the perceived length of time in the waiting area were solicited. All responses were measured on a four point Likert scale. Minors, adults who did not read or speak English, and caretakers accompanying children arriving in the department outside the usual triage process were excluded. Results: A study group totaled of 295 participants with an average age of 32 years, $61 \%$ female. Two-thirds identified themselves as a parent of a patient. The mean anxiety level was 41 . There was no difference in mean anxiety levels according to gender, age, or relationship to the patient. The mean anxiety level for those with perceived wait times over one hour was significantly higher than those with wait times less than one hour. (49.9. vs 38.9, p .006). Factors which may contribute to stress were elicited using a 4 point Likert scale. Worry over the child's illness (mean 2.76) and perceived long wait times (mean 2.56) were the most highly ranked causes of stress in the ED, as and perceived long wait times (mean 2.56) were the most highly ranked causes of stress in the ED, as
compared to environmental factors, which ranged from 1.32-2.00. Conclusion: Long wait times are associated with significantly increased anxiety among adults accompanying children to the ED. Worry over the child's illness and perceived long wait times were the most influential factors related to causes of stress in the ED, while environmental factors were considered less important. Interventions to minimize perceived wait times and worry over the child's illness may result in diminished anxiety for adults waiting with their child in an ED.

\section{3}

WHAT INFLUENCES PARENTS' DECISIONS TO LIMIT OR WITHDRAW LIFE SUPPORT?

M Sharma, KL Meert, AP Sarnaik, Department of Pediatrics, Division of Critical Care Medicine, Children's Hospital of Michigan, Wayne State University, Detroit, MI.

Purpose: Decisions to limit/withdraw life support from critically ill children are commonly faced by parents and physicians. Previous research regarding parents' perspectives on end-of-life (EOL) decision-making in the PICU has been limited by retrospective methods and the use of closed-ended questions with predetermined response choices. Deeper understanding of parents' views will allow physicians to focus EOL discussions on factors important to parents and help to resolve conflicts. We prospectively identified and described parents' self-reported influences on EOL decision-making for critically ill children. Methods: Parents of children whose PICU physician had made a recommendation to limit/withdraw life support from their child were eligible for the study. Parents participated in semi-structured audio taped interviews regarding their decision-making process. Interviews were conducted during the child's PICU stay. Interviews were transcribed verbatim and independently coded by two investigators to identify segments of text that describe factors influencing parents' EOL decisions. Results: Fourteen parents ( 9 mothers, 5 fathers) of 10 children were interviewed during the decision-making process. Factors influencing parents' decisions to limit/withdraw support included their personal observation of their child's suffering and decline; previous experience with death/EOL decisions; perceptions of their child's will-to-survive regardless of the child's cognitive and developmental state; their child's previous response to critical care interventions; their need to protect and advocate for their child; and the family's financial resources and concerns regarding life-long care. Parents expressed an overwhelming desire to do what is best for their child but struggled with feelings of selfishness, guilt and the need to avoid agony and sorrow. Physician recommendations, review of options and joint formulation of a plan helped parents gain a sense of control over their situation Parents of eight children agreed to limit/withdraw life support and parents of two did not. Conclusions: Prospective interviews with open-ended questions identified factors influencing parents' EOL decisions such as their past experience with death and their anticipated emotional adjustments and future resources not previously described in the critical care literature. Inclusion of these factors into discussions with parents may facilitate the decision-making process. More prospective research is needed to further understand what is meaningful to parents during EOL decision-making for children.

\section{4}

NON-INVASIVE CONTRAST ENHANCED ELECTRON BEAM TOMOGRAPHY: A THREE DIMENSION IMAGING ADJUNCTIVE TEST TO DETECT RENAL VASCULAR AND A-V FISTULA GRAFT ABNORMALITIES.

EG John, A Lumpaopong, C Ruiz, Department of Pediatrics, University of Illinois at Chicago, Chicago, IL.

Electron beam tomography (EBCT) is a relatively new technology with several potential advantages over traditional CT such as fast acquisition time, less exposure to radiation, less sedation and volume of dye, and better 3D visualization. A retrospective study was done to assess the use of 3D EBCT to detect renal vascular abnormalities and patency of arteriovenous-fistula (A-VF) and arteriovenousgraft (A-VG). EBCT was done in 7 children (age 3-18 year, mean age 10.8 years). All had hypertension $(>95 \%$ ) and high rennin levels. Four of the patients (group I) had normal renal function. One patient (group II) was 20 days post enblock renal transplantation with ascites, hypertension and renal dysfunction. Two patients (group III) had end-stage renal disease, hypertension, and AV-F and AV-G. One of these patients had SLE and difficult IV access. In group I, 2/4 had left ventricular hypertrophy, eye and CNS changes. MAG-3 renal scan (RSC) and renal ultrasound (RUS) were normal in $2 / 4$ group I patients. In group I, $2 / 4$ EBCT showed unilateral renal artery stenosis: one of these children had decreased velocity in one kidney by RUS and the other child had possible renal artery stenosis by RSC. One child in group I with abnormal RUS suggestive of RAS did not have RAS by EBCT and traditional angiography. In group II, patient with transplant, EBCT showed normal renal vasculature and ureterovesicle anastomoses without RAS or leak respectively. In group III, the SLE patient had thrombosis of the inferior vena cava and renal veins and the other patient had patent A-VF with dilated vessels contrary to US finding of thrombosis. In conclusion, EBCT with its non invasive, maximum intensity projection and 3D images, less radiation and acquisition time is a useful adjunct test to detect RAS and AV-F, AV-G and transplant vascular thrombosis/stenosis in children. 